\title{
Model Selection based on the Angular-Diameter Distance to the Compact Structure in Radio Quasars
}

\author{
F. MELIA ${ }^{1}$ \\ 1 Department of Physics, The Applied Math Program, and Department of Astronomy, \\ The University of Arizona, AZ 85721, USA
}

\author{
PACS 95.36.+x - Dark Energy \\ PACS 98.80.-k- Cosmology \\ PACS 98.80. Jk - Relativistic Astrophysics
}

\begin{abstract}
Of all the distance and temporal measures in cosmology, the angular-diameter distance, $d_{A}(z)$, uniquely reaches a maximum value at some finite redshift $z_{\max }$ and then decreases to zero towards the big bang. This effect has been difficult to observe due to a lack of reliable, standard rulers, though refinements to the identification of the compact structure in radio quasars may have overcome this deficiency. In this Letter, we assemble a catalog of 140 such sources with $0 \lesssim z \lesssim 3$ for model selection and the measurement of $z_{\max }$. In flat $\Lambda \mathrm{CDM}$, we find that $\Omega_{\mathrm{m}}=0.24_{-0.09}^{+0.1}$, fully consistent with Planck, with $z_{\max }=1.69$. Both of these values are associated with a $d_{A}(z)$ indistinguishable from that predicted by the zero active mass condition, $\rho+3 p=0$, in terms of the total pressure $p$ and total energy density $\rho$ of the cosmic fluid. An expansion driven by this constraint, known as the $R_{\mathrm{h}}=c t$ universe, has $z_{\max }=1.718$, which differs from the measured value by less than $\sim 1.6 \%$. Indeed, the Bayes Information Criterion favours $R_{\mathrm{h}}=c t$ over flat $\Lambda \mathrm{CDM}$ with a likelihood of $\sim 81 \%$ versus $19 \%$, suggesting that the optimized parameters in Planck $\Lambda$ CDM mimic the constraint $p=-\rho / 3$.
\end{abstract}

Introduction. - The luminosity distance is used often in cosmology for measurements involving standard candles, such as Type Ia SNe [1] and gamma ray bursts [2]. By comparison, the angular-diameter distance, $d_{A}(z)$, applicable to objects whose diameter (preferably a 'standard ruler') is known, is used only sparingly, given the relative paucity of such sources and complications arising from size evolution with redshift. More typically, sources used to measure $d_{A}(z)$ have been restricted to narrow ranges in redshift, mitigating their possible impact on revealing the geometric structure of the Universe over large distances. The cases where some progress has been made with the use of $d_{A}(z)$ include (1) the use of baryon acoustic oscillations seen in large-scale structure $[3,4] ;(2)$ the Sachs-Wolfe induced $\sim 10^{\circ}$ fluctuations seen in the cosmic microwave background [5-7]; (3) strong lensing systems, with and without time delays [8-12]; and (4) galaxy clusters [13-15]. But several developments in our understanding of the compact structure in radio quasars have presented us with what appears to be a more reliable measuring rod, whose negligible evolution in the redshift range $0 \lesssim z \lesssim 3$ permits precision cosmological testing over a larger fraction of the Universe's age than is feasible with these other methods, or even through the measurement of the luminosity distance using Type Ia SNe. As we shall see below, these developments follow primarily from our improved understanding of synchrotron self-absorption processes near the central engine of active galactic nuclei (see, e.g., refs. [16,17] and references cited therein) and the identification of critical constraints on the observed characteristics of these sources - principally their spectral index and luminosity - that permit the selection of an appropriate sample with a more or less fixed size of the emission region for cosmological testing [18-21].

Measuring the geometry of the Universe with standard rulers was first proposed by Hoyle [22] over half a century ago, though it took several decades before attempts were made to actually implement this proposal using actual sources. And the earliest tests of cosmological models based on the observed redshift dependence of the angular size of kpc-scale radio sources and galaxies were not successful due to the lack of a reliable, well-defined standard ruler [23-28]. Some motivation to continue pursuing this quest finally came with a study of double-lobed quasars 
within the redshift range $1.0 \lesssim z \lesssim 2.7$, which showed no change in apparent angular size with angular-diameter distance, somewhat consistent with Friedmann-RobertsonWalker cosmology without any significant evolution (see fig. 1 below) [29].

It eventually became apparent that ultracompact radio sources are more likely to produce standard measuring rods than the large-scale jets in quasars and radio galaxies. The emission from these compact regions is dominated by self-absorbed synchrotron emission [16], forming at least partially opaque features with angular diameters in the milliarcsecond (mas) range, and linear sizes of the order of 10 parsecs $[17,30]$. Their significant advantage over larger structures, such as galaxies and kpc-scale jets, is that these central cores are much smaller than their parent active galactic nuclei (AGN), so their ambient physical environment should be similar from source to source and be reasonably stable, unlike the variations one expects in the intergalactic medium over large distances and times $[31,32]$. The compact structures in these sources therefore evolve principally under the influence of the central engine itself, which is typically characterized by only a few physical parameters, such as the mass of the black hole and its spin. Dynamical timescales in such environments are only tens of years, much shorter than the age of the Universe. The compact structures in radio quasars should therefore be free of long-term evolutionary effects [18].

In one of the more significant studies involving the compact structure of radio quasars, Gurvits, Kellermann \& Frey [18] showed that a large sample of images taken with Very Large Baseline Interferometry (VLBI) may be used to establish some general constraints on cosmological parameters. This work has formed the basis of many subsequent investigations [33-35], leading to a second significant advancement with the use of these sources that we shall discuss shortly [20].

A persistent complication with compact radio jets has been that they are found in a mixed population of radio galaxies and AGNs - quasars, BL Lacs, OVVs, etc.-so systematic differences are not always easy to disentangle from true cosmological variations. Recent work by Cao et al. [19], however, has included the analysis of different AGN sub-samples based on different source optical counterparts and lying in different redshift ranges, leading to the conclusion that radio galaxies and quasars need to be handled with distinct strategies. This result is the basis for the current study of such sources, focusing only on compact structures in radio quasars $[20,21]$. The net outcome of this effort has been a significant reduction in scatter to produce a reliable sample of compact structures in radio quasars for use as standard rulers, allowing us to carry out the study reported in this Letter, which compares models in ways not previously feasible with other measures of cosmological distance.

Data and Analysis. - Following the suggestion by Gurvits et al. [18] and Vishwakarma [37] that the exclusion of sources with low luminosities $L$ and extreme spectral indices, $\alpha$, might curb the dependence of the core size on the source luminosity and redshift, several workers have refined the process of compiling from the many hundreds of available VLBI images a reduced sample with manageable scatter, free of evolutionary effects.

With very long baseline interferometry, the signal from a distant radio source is received at multiple radio telescopes across Earth's surface, whose registration of intensities may then be correlated taking into account the slightly different arrival times at the various facilities. The net result is a combined observation made by a telescope with a baseline equal to the maximum separation of the radio antennae. For the compact structure in radio quasars, the characteristic angular size inferred from VLBI is defined as

$$
\theta_{\text {core }} \equiv \frac{2 \sqrt{-\ln \Gamma \ln 2}}{\pi B},
$$

where $B$ is the interferometer baseline and $\Gamma$ is the ratio of total flux density to the correlated flux density [39]. The linear size of the core may then be written

$$
\ell_{\text {core }}=\theta_{\text {core }}(z) \times d_{A}(z) \text {, }
$$

where $d_{A}(z)$ is the model-dependent angular-diameter distance.

It is now understood that the dispersion in linear size is greatly mitigated $[18,20]$ by retaining only those sources with $-0.38<\alpha<0.18$. Additionally, Cao et al. [20] have recently pointed to a strong dependence of the core size $\ell_{\text {core }}$ on luminosity, not just at the low end, but at the high end as well. Using the parametrization $\ell_{\text {core }}=$ $\ell_{0} L^{\beta}(1+z)^{n}$, where $\ell_{0}$ is a scaling constant, they demonstrated that only a sub-sample of intermediate-luminosity radio quasars $\left(10^{27} \mathrm{~W} / \mathrm{Hz}<L<10^{28} \mathrm{~W} / \mathrm{Hz}\right)$ have a core size with negligible dependence on $L$ and $z$. For these objects, $\beta \approx 10^{-4}$ and $|n| \approx 10^{-3}$, yielding a compilation of compact structures in radio quasars with a rather robust standard linear size.

The data we use here were assembled by Jackson \& Jannetta [38] using the $2.29 \mathrm{GHz}$ VLBI survey of Preston et al. [39] and additions by Gurvits [40], resulting in a catalog of 613 sources. In order for us to extract the subsample with luminosities restricted to the range alluded to above, we use the Planck optimized parameters [41] to estimate the luminosity distance and thereby the value of $L$ from the measured total flux density at $2.29 \mathrm{GHz}$. These parameters are used merely to estimate $L$ for the purpose of identifying the intermediate-luminosity sources, and are not otherwise employed in the fitting procedure described below. We have carried out a simple test to ensure that the model selection is not biased with this approach by relaxing the constraint on $L$ by as much as $50 \%$, which produced no discernable effect. In addition, note that using the Planck parameters would benefit $\Lambda$ CDM, if at all, so an outcome favouring $R_{\mathrm{h}}=c t$ could not be viewed as having been facilitated by this approximation. The sample 


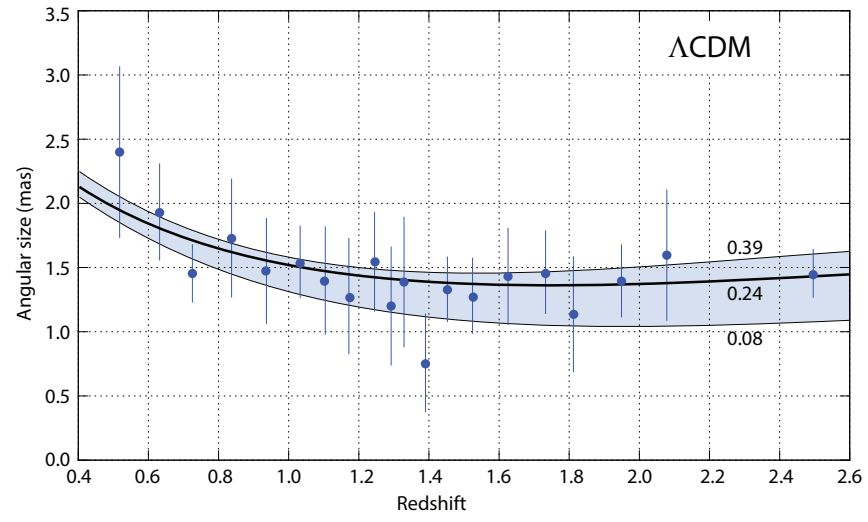

Fig. 1: Angular size of 140 individual compact structures in radio quasars binned into groups of 7 , as a function of redshift. The data points represent the median value in each bin. The thick solid curve is the optimized flat $\Lambda \mathrm{CDM}$ model, with angular size constant $\eta=0.58 \pm 0.05$ and $\Omega_{\mathrm{m}}=0.24_{-0.09}^{+0.1}$ (see text). Also shown are the values of $\Omega_{\mathrm{m}}$ (i.e., 0.08 and 0.39 ) setting the bounds of the $1 \sigma$ shaded region (when $\eta$ is held constant)

is further reduced by restricting $\alpha$ to the aforementioned range of spectral indices, producing a final subsample of 140 sources for our study. These are binned into groups of 7 and, following previously established convention [42], we select the median value to represent the angular size in each bin. These data are plotted in figure 1 , together with their $1 \sigma$ errors estimated assuming a Gaussian distributed variation in each bin.

Using the angular-diameter distance with these sources allows us to study the geometry of the Universe in several unique ways. First, as long as $\ell_{\text {core }}$ is a true standard ruler, at least in an average sense, we do not need to know its actual value for model selection because we are merely sampling the ratio of scales at different redshifts. For the same reason, we also do not need to know the Hubble constant, $H_{0}$, further reducing the number of model parameters that need to be optimized. And unlike Type Ia $\mathrm{SNe}$, which may be detected only up to $z \sim 1.8$ [1], compact radio jets have been mapped with VLBI as far out as $z \sim 4$ [38-40]. In principle, we may therefore examine the geometry of the Universe over almost $80 \%$ of its existence, compared to smaller fractions with other techniques. This follows from the fact that, in flat $\Lambda \mathrm{CDM}$, a redshift of 2.6 corresponds to an age of about $2.5 \mathrm{Gyr}$, which represents a look back fraction $1-2.5 / 13.7 \approx 0.82$, using today's age of $\sim 13.7$ Gyr. Most critically, among the various distance measures in cosmology, $d_{A}(z)$ alone has a maximum value at a redshift $z_{\max }$ that varies from one model to the next. The angular-diameter distance increases at first, peaks at $z_{\max }$, and then decreases at higher redshifts, reaching zero at the big bang. It is not difficult to understand why this happens [43]. When we measure a lateral proper size, we see the object as it was when it emitted the light reaching us today, and since all sources were closer to us as we

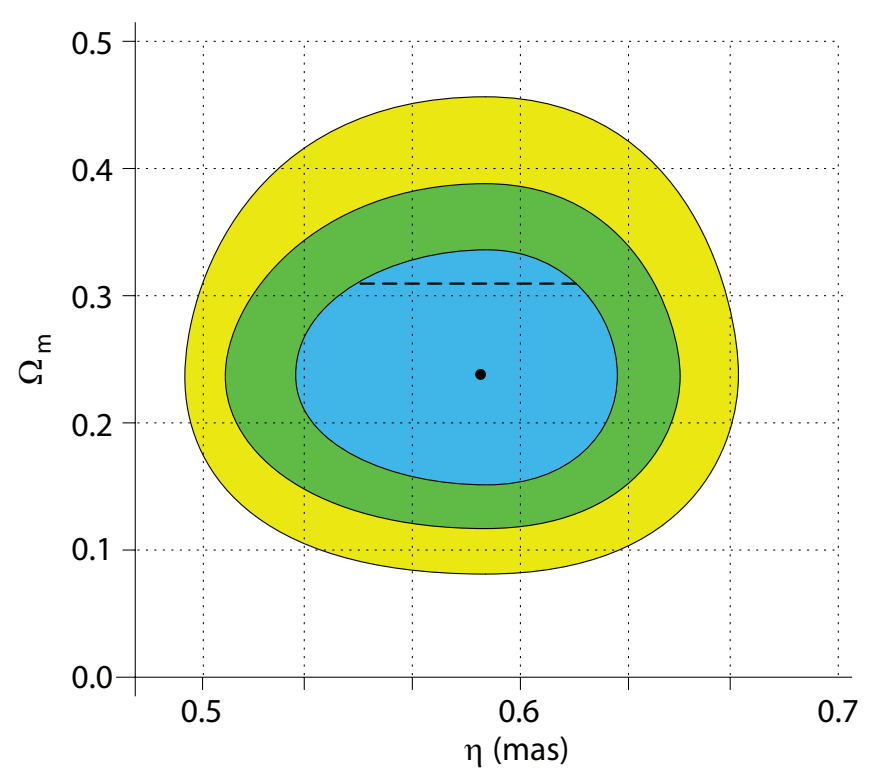

Fig. 2: One (blue), two (green), and three (yellow) $\sigma$ confidence regions associated with the optimized parameters $\Omega_{\mathrm{m}}$ and $\eta$ in flat $\Lambda$ CDM (see Table 1). By comparison, the horizontal dashed line shows the Planck [41] value $\Omega_{\mathrm{m}}=0.308$, which lies within $1 \sigma$ of the measurement based on compact structures in radio quasars.

look back in time, their apparent angular size $\theta_{\text {core }}$ actually gets bigger as $z \rightarrow \infty$, with the compensating effect that $d_{A}(z)\left(\sim \theta_{\text {core }}^{-1}\right)$ therefore gets smaller.

We will keep this analysis as simple and parameterfree as possible, concentrating solely on what is absolutely needed in order for us to extract information concerning the geometry of the Universe from the compact radio-jet data shown in figure 1 . For a given core size $\ell_{\text {core }}$, the predicted angular size of the compact structure in radio quasars is obtained from Equation (2), in terms of the angular-diameter distance which, in flat $\Lambda \mathrm{CDM}$, is given as

$$
\begin{gathered}
d_{A}(z)=\frac{c}{H_{0}} \frac{1}{1+z} \times \\
\int_{0}^{z} \frac{d u}{\left[\Omega_{\mathrm{m}}(1+u)^{3}+\Omega_{\mathrm{r}}(1+u)^{4}+\Omega_{\Lambda}(1+u)^{3+3 w_{\Lambda}}\right]^{1 / 2}}
\end{gathered}
$$

In this expression, $\Omega_{i}$ is the energy density of species " $i$ " scaled to today's critical density, $3 c^{2} H_{0}^{2} / 8 \pi G$, and $w_{\Lambda}=-1$ is the equation-of-state parameter for a cosmological constant. Given that radiation energy density is relatively negligible up to $z \sim 3$, there are really only two free parameters in the expression for $\theta_{\text {core }}$ once we marginalize over the unknowns $\ell_{\text {core }}$ and $H_{0}$. We do this by combining Equations (2) and (3) and writing

$$
\theta_{\text {core }}(z)=\eta \frac{1+z}{\mathcal{I}(z)}
$$


where $\eta \equiv \ell_{\text {core }} H_{0} / c$ and

$$
\mathcal{I}(z)=\int_{0}^{z} \frac{d u}{\left[\Omega_{\mathrm{m}}(1+u)^{3}+1-\Omega_{\mathrm{m}}\right]^{1 / 2}} .
$$

Using standard $\chi^{2}$ minimization, we optimize the values of $\eta$ and $\Omega_{\mathrm{m}}$ that produce the best fit, shown as a solid, black curve in figure 1 . The results of this fitting are summarized in Table 1 , and the corresponding $1 \sigma, 2 \sigma$ and $3 \sigma$ confidence regions are plotted on the $\eta-\Omega_{\mathrm{m}}$ plane in figure 2. To further emphasize the quality of the fit, we also show in figure 1 the $1 \sigma$ confidence region estimated by keeping $\eta$ fixed and allowing $\Omega_{\mathrm{m}}$ to vary. The curves bounding this confidence region correspond to $\Omega_{\mathrm{m}}=0.08$ and 0.39 . We therefore find that the value of $\Omega_{\mathrm{m}}$ optimized with fits to the compact radio-jet data is fully consistent with Planck $\Lambda$ CDM at a level of confidence of better than $1 \sigma$.

Discussion. - Let us now turn our attention to the primary goal of using these sources for model selection purposes based solely on the inferred geometry of the Universe. The turning point in the fitted $\theta_{\text {core }}(z)$ function is a byproduct of $d_{A}(z)$ 's unique maximum value at a modeldependent redshift $z_{\max }$. The best-fit curve in figure 1 has a turning point at $z_{\max }=1.69$, with a possible variation between 1.42 and 2.20 across the $1 \sigma$ confidence region, a swing of about $46 \%$ from top to bottom.

We wish to understand what is so special about the values $\Omega_{\mathrm{m}}=0.24$ and $z_{\max }=1.69$ that the Universe would have 'chosen' these to characterize its geometry and expansion. Such questions have been asked repeatedly over the past several decades as the analysis of each new dataset has unfolded. What has emerged is an indication that $\Lambda \mathrm{CDM}$ may be lacking an additional constraint on its constituents that may resolve the origin of these parameter values.

$\Lambda \mathrm{CDM}$ adopts the equation of state $p=w \rho$, with $p=p_{\mathrm{m}}+p_{\mathrm{r}}+p_{\mathrm{de}}$ and $\rho=\rho_{\mathrm{m}}+\rho_{\mathrm{r}}+\rho_{\mathrm{de}}$, where $p_{i}$ and $\rho_{i}$ are the pressure and energy density, respectively, of species " $i$ " in the cosmic fluid, with " $\mathrm{m}$ " representing (luminous and dark) matter, "r" radiation and "de" dark energy. The latter may or may not be a cosmological constant. The expansion dynamics is based on the assumption that $p_{\mathrm{m}}=0$, $p_{\mathrm{r}}=\rho_{\mathrm{r}} / 3$ and (typically) $p_{\mathrm{de}}=-\rho_{\mathrm{de}}$, while the densities $\rho_{\mathrm{m}} \sim(1+z)^{-3}, \rho_{\mathrm{r}} \sim(1+z)^{-4}$ and $\rho_{\text {de }} \sim(1+z)^{0}$ each evolve with redshift independently of the others. But the data appear to be telling us something different, pointing to a coupling of the densities in order to preserve a constant equation-of-state with $w=-1 / 3$, known as the 'zero active mass' condition in general relativity. In test after test, the predictions of $\Lambda$ CDM with this additional constraint, a model referred to as the $R_{\mathrm{h}}=c t$ Universe [44-48] in the literature, have been a better match to the data than those of basic $\Lambda$ CDM without it. These comparisons have been carried out using a broad range of observations, from the angular correlation function of the cosmic microwave background [49] and high- $z$ quasars $[50,59]$ in

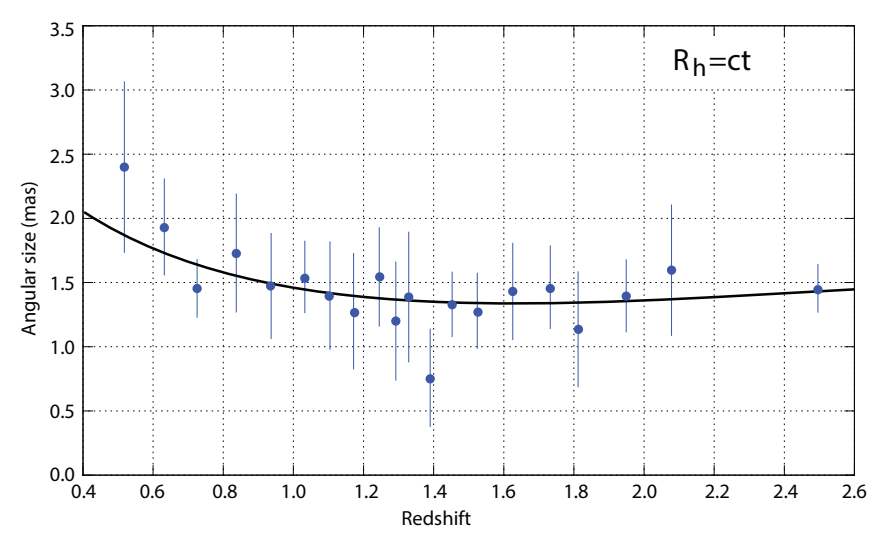

Fig. 3: Same as figure 1, except now comparing the data with the curve predicted by the $R_{\mathrm{h}}=c t$ Universe. Once the Hubble constant $H_{0}$ and the size of the compact structure are marginalized, there are no free parameters left with which to optimize the fit. Even without this flexibility, however, this curve accounts for the data better than $\Lambda$ CDM (see Table 1).

the early Universe, to gamma ray bursts [52] and cosmic chronometers [53] at intermediate redshifts, and to the relatively nearby Type Ia SNe [54]. A recently compiled list of these comparative studies may be found in Table 1 of [55].

The angular-size data presented in figure 1 allows us examine this growing body of evidence from an entirely different perspective, because we have never had an opportunity before the identification of this sample of compact radio structures by key workers in this field, including Gurvits [40], Jackson [32], Cao et al. [20,21] and others, of testing the prediction that $d_{A}(z)$ ought to have a maximum at a finite redshift $z_{\max }$. When we impose the additional constraint $w=-1 / 3$ on $\Lambda \mathrm{CDM}$, Equation (5) is simply

$$
\mathcal{I}(z)=\ln (1+z) .
$$

The angular size of the compact radio jets predicted by this model is now shown together with the data in figure 3. It is virtually indistinguishable from the best-fit $\Lambda \mathrm{CDM}$ curve in figure 1 . Indeed, the reduced $\chi_{\text {dof }}^{2}$ 's for these two fits are identical (see Table 1). But what is particularly significant is the fact that the theoretical curve in figure 3 has no free parameters (other than the 'nuisance' variable $\eta$, which is common to both models). In model selection, one must take the different number of free parameters into account when evaluating relative likelihoods. In cosmology, this is now routinely done using information criteria, such as the Bayes Information Criterion (BIC) applicable to large samples [53], defined as $\mathrm{BIC}=\chi^{2}+k \ln n$, where $k$ is the number of free parameters and $n$ is the sample size. In a head to head comparison between two models, the BIC yields the relative probability of either being 'closer' to the truth and, as one can see from the outcomes listed in Table 1 , the use of $d_{A}(z)$ with the compact radio-jet data favours $R_{\mathrm{h}}=c t$ over $\Lambda$ CDM.

We are therefore seeing a strong confirmation of previ- 
Table 1: Model Selection Using the Compact Structure of Radio Quasars

\begin{tabular}{cccccc} 
Model & $\Omega_{\mathrm{m}}$ & $\eta$ & $\chi_{\text {dof }}^{2}$ & BIC & Probability \\
\hline \hline$\Lambda \mathrm{CDM}$ & $0.24_{-0.09}^{+0.1}$ & $0.58 \pm 0.05$ & 0.31 & 11.6 & $19.8 \%$ \\
$R_{h}=c t$ & - & $0.5_{-0.02}^{+0.03}$ & 0.31 & 8.8 & $80.2 \%$ \\
\hline \hline
\end{tabular}

ous results based on other kinds of measurement. This outcome suggests that the optimized value of $\Omega_{\mathrm{m}}$ in $\Lambda \mathrm{CDM}$ arises because the formulation of $w$ in this model needs it to mimic the integral in Equation (6) associated with the zero active mass condition $w=-1 / 3$. But the most convincing evidence in support of this conclusion comes from an evaluation of $z_{\max }$ in $R_{\mathrm{h}}=c t$, a new probe of the Universe's geometry - never seen before the recent work of Cao et al. [20,21] with any other kind of measurement. From Equations (4) and (6) one finds that $z_{\max }=1.718$ when the zero active mass condition is imposed on $\Lambda \mathrm{CDM}$. This value lies within $1.6 \%$ of the turning point in $\theta_{\text {core }}$ found with the formulation of $w$ in the standard model suggesting, once again, that the measured value of $z_{\max }$ is not random at all, but is a direct consequence of the Universe's expansion at a rate consistent with the zero active mass condition.

Finally, let us consider what the optimized value of $\eta$ in Table 1 implies for the Hubble constant $H_{0}$, should the core size $\ell_{\text {core }}$ be known from other data. A difficulty generally arises in the optimization of $H_{0}$ due to its model dependence. There is no universal way of measuring its value without assuming a particular model. The Hubble constant has been measured locally, e.g., using Cepheid variables, though it disagrees with the Planck value by more than $9 \%$. It is not yet clear why this happens, but some have speculated that a local "Hubble bubble" (Shi 1997; Keenan et al. 2013; Romano 2017) may be influencing the local dynamics within a distance $\sim 300 \mathrm{Mpc}$ (i.e., $z \lesssim 0.07$ ). If true, such a fluctuation might lead to anomalous velocities within this region, causing the nearby expansion to deviate somewhat from a pure Hubble flow. For consistency, $H_{0}$ must therefore be measured on large, smoothed scales.

With our value of $\eta, H_{0}$ may be inferred once $\ell_{\text {core }}$ is known. An estimate of its value was made recently by ref. [21], who used measurements of the expansion rate $H(z)$ based on cosmic chronometers to break the degeneracy between $\ell_{\text {core }}$ and the Hubble constant. Their analysis estimated the core size to be $\ell_{\text {core }} \sim 11.03 \pm 0.25$ pc. Thus, for the optimized value $\eta=0.5_{-0.02}^{+0.03}$ in $R_{\mathrm{h}}=c t$ (see Table 1), the implied Hubble constant is $H_{0}=66.0_{-2.6}^{+4.0} \mathrm{~km}$ $\mathrm{s}^{-1} \mathrm{Mpc}^{-1}$. As of today, $H_{0}$ in $R_{\mathrm{h}}=c t$ has been measured 4 times: $63.2 \pm 1.6 \mathrm{~km} \mathrm{~s}^{-1} \mathrm{Mpc}^{-1}$ [53], $63.3 \pm 7.7 \mathrm{~km} \mathrm{~s}^{-1}$ $\mathrm{Mpc}^{-1}[59], 62.3 \pm 1.5 \mathrm{~km} \mathrm{~s}^{-1} \mathrm{Mpc}^{-1}$ [60] and $63.0 \pm 2.0$ $\mathrm{km} \mathrm{s}^{-1} \mathrm{Mpc}^{-1}$ [61]. Cao et al.'s estimate of $\ell_{\text {core }}$ therefore yields a Hubble constant $H_{0}$ for $R_{\mathrm{h}}=c t$ consistent with these previous measurements.

The corresponding Hubble constant in $\Lambda$ CDM is $76.6 \pm$ $6.6 \mathrm{~km} \mathrm{~s}^{-1} \mathrm{Mpc}^{-1}$. But while this value is still consistent with the Planck optimization, it is nonetheless somewhat on the high side, in slight tension with the estimates made in ref. [21]. It is very instructive to examine the cause of this non-trivial difference. There are actually two principal reasons why our inferred value of $H_{0}$ for $\Lambda$ CDM does not agree completely with that obtained earlier by ref. [21].

First, while Cao et al. carried out a $\chi^{2}$-minimization using the individual quasars in our sample of 120 , our approach calls for the binning of these sources into 20 redshift intervals before optimizing the model parameters. A quick inspection of their data and best fit curves shows that many of the individual sources lie several $\sigma$ 's away from the theoretical curves. In other words, the reported errors are far too small to represent the actual scatter in the data. For this reason, we have chosen to bin the individual sources and use population variance based on assumed Gaussian variation within each bin to more reliably estimate the error associated with each data point. Not surprisingly, our errors are larger than those reported for each individual source because they better reflect the overall scatter in the data. A quick inspection of figs. 1 and 3 shows that all but one of the data in these plots lie within $1 \sigma$ of the best-fit curves. We suggest that carrying out a model optimization with these binned data is therefore more reliable than simply trying to do this with data whose errors are unrealistically small.

Second, and more importantly, Cao et al. [21] optimized $\ell_{\text {core }}$ and $H_{0}$ separately, while (as noted above), we optimize the sole parameter $\eta$. Why is this important? Cao et al. did not base their parameter optimization solely on the quasar-core data. Since they needed additional information to separate these two unknowns, they combined their angular-size measurements with observations of $H(z)$ based on cosmic chronometers, as noted earlier. As such, their optimized parameters reflect the joint analysis of several different data sets, as opposed to just the quasar-core observations - the principal focus in this paper. Each approach has its advantages, of course. Ours allows model selection to be carried out based solely on the quasar-core data. This is not a trivial step, because each kind of measurement should be studied on its own, not only in joint analyses with other observations that may introduce unknown biases.

Conclusion. - Needless to say, the identification of the compact structure in radio quasars as standard rulers has opened up an entirely new chapter in cosmology. With them, we may now map the geometry of the Universe well beyond the reach of Type Ia SNe, sampling even the epoch during which the apparent size of sources increases with redshift, an effect not seen with any other kind of measurement probe. The results thus far point to the zero 
active mass condition in general relativity as the influence guiding the Universe's expansion. Developing this notion further, and testing it with even higher precision measurements, promises a very exciting future in observational cosmology.

\section{$* * *$}

I am very grateful to the anonymous referee, who has provided many suggestions for improving the presentation in this paper. I am also grateful to Amherst College for its support through a John Woodruff Simpson Lectureship, and to Purple Mountain Observatory in Nanjing, China, for its hospitality while part of this work was being carried out. This work was partially supported by grant 2012T1J0011 from The Chinese Academy of Sciences Visiting Professorships for Senior International Scientists, and grant GDJ20120491013 from the Chinese State Administration of Foreign Experts Affairs.

\section{REFERENCES}

[1] Kowalski, M. et AL., Astrophys. J., 686 (2008) 749.

[2] Wei, J.-J., Wu, X. And Melia, F., Astrophys. J., 772 (2013) 43.

[3] Melia, F. And López-Corredoira, M., Int. J. Mod. Phys. D, 26 (2017) 1750055.

[4] Alcock, C. and Paczyński, B., Nature, 281 (1979) 358.

[5] Bennett C. et AL., Astrophys. J. Suppl. Ser., 148 (2003) 97.

[6] Spergel, D. N. et Al., Astrophys. J. Suppl. Ser., 148 (2003) 175.

[7] Melia, F. And López-Corredoira, M., Astron. Astrophys., 610 (2018) A87.

[8] Refsdal, S., Mon. Not. R. Soc., 128 (1964) 307.

[9] Treu, T. ET AL., Astrophys. J., 640 (2006) 662.

[10] Biesiada, M. et Al., Mon. Not. R. Soc., 406 (2010) 1055.

[11] Wei, J.-J., Wu, X. And Melia, F., Astrophys. J., 788 (2014) 190.

[12] Melia, F., Wei, J.-J. And Wu, X., Astron. J., 149 (2015) 2.

[13] Sasaki, S, Pub. Astron. Soc. Japan, 48 (1996) L119.

[14] Pen, U.-L., New Astron., 2 (1997) 309.

[15] Melia, F., Proc R Soc A, 472 (2016) 20150765.

[16] Blandford, R. D. And Königl, A., Astrophys. J., 232 (1979) 34.

[17] High-Energy Astrophysics (Princeton University Press) 2009.

[18] Gurvits, L. I., Kellermann, K. I. \& Frey, S., Astron. Astrophys., 342 (1999) 378.

[19] CaO, S. et Al., Astrophys. J., 806 (2015) 66.

[20] CaO, S. ET AL., J. Cosm. Astropart. Phys., 02 (2017) 012.

[21] CaO, S. ET AL., Astron. Astrophys., 606 (2017) A15.

[22] Bracewell, R. N. (Editor), Paris Symposium on Radio Astronomy, IAU Symp. 9, URSI Symp. 1, Vol. 9 (Stanford University Press) 1959.

[23] Sandage, A. R., Ann. Rev. Astron. Astrophys., 26 (1988) 56.
[24] Hewitt, A., Burbidge, G. And Fang, L. Z. (Editor), Observational Cosmology (IAU Symp. 124), Vol. 124 (Reidel) 1987

[25] Barthel, P. D. \& Miley, G. K., Nature, 333 (1988) 319.

[26] Neeser, M. J. ET Al., Astrophys. J., 451 (1995) 76.

[27] Singal, A. K., Mon. Not. R. Soc., 263 (1993) 139.

[28] Nilsson, K. ET AL., Astrophys. J., 413 (1993) 453.

[29] Buchalter, A. et Al., Astrophys. J., 494 (1998) 503.

[30] Kellerman, K. I. and Pauliny-Toth, K.I.A., Ann. Rev. Astron. Astrophys., 19 (1981) 373.

[31] Kellermann, K. I., Nature, 361 (1993) 134.

[32] Jackson, J. C., Mon. Not. R. Soc., 390 (2008) L1.

[33] Lima, J.A.S. \& Alcaniz, J. S., Astrophys. J., 566 (2002) 15.

[34] Zhu, Z.-H. \& Fujimoto, M. K., Astrophys. J., 581 (2002) 1 .

[35] Chen, G. \& Ratra, B., Astrophys. J., 582 (2003) 586.

[36] Preston, R. A., Morabito, D. D., Williams, J. G. ET AL., Astron. J., 90 (1985) 1599.

[37] Vishwakarma, R. G., Class. Quantum Grav., 18 (2001) 1159.

[38] Jackson, J. C. \& Jannetta, A. L., J. Cosm. Astropart. Phys., 11 (2006) 002.

[39] Preston, R. A., Astron. J., 90 (1985) 1599.

[40] Gurvits, L. I., Astrophys. J., 425 (1994) 442.

[41] Planck Collaboration et Al., Astron. Astrophys., 594 (2016) A13.

[42] Santos, R. C. \& Lima, J.A.S., Phys. Rev. D, 77 (2008) 083505 .

[43] Melia, F., Class Quantum Grav., 30 (2013) 155007.

[44] Melia, F., Mon. Not. R. Soc., 382 (2007) 1917.

[45] Melia, F. \& Abdelqader, M., Int. J. Mod. Phys. D, 18 (2009) 1889.

[46] Melia, F. \& Shevchuk, A.S.H., Mon. Not. R. Soc., 419 (2012) 2579.

[47] Melia, F., Frontiers of Physics, 11 (2016) 119801.

[48] Melia, F., Frontiers of Physics, 12 (2017) 129802.

[49] Melia, F., Astron. Astrophys., 561 (2014) A80.

[50] Melia, F., Astrophys. J., 764 (2013) 72.

[51] Melia, F. \& McClintock, T. M., Proc. R. Soc. A, 471 (2015) 20150449.

[52] Wei, J.-J., Wu, X., Melia, F., Wei, D. M. \& Feng, L. L., Mon. Not. R. Soc., 439 (2014) 3329.

[53] Melia, F. \& Maier, R., Mon. Not. R. Soc., 432 (2013) 2669.

[54] Wei, J.-J., Wu, X., Melia, F. \& Maier, R. S., Astron. J., 149 (2015) 102.

[55] Melia, F., Mon. Not. R. Soc., 464 (2017) 1966.

[56] ShI, X., Astrophys. J., 486 (1997) 32.

[57] Keenan, R. C. ET AL., Astrophys. J., 775 (2013) 62.

[58] Romano, A. E., e-print, (2017) arXiv:1609.04081

[59] Melia, F. \& McClintock, T. M., Astron. J., 150 (2015) 119.

[60] Wei, J.-J., Melia, F. \& Wu, X., Astrophys. J., 835 (2017) 270.

[61] Melia, F. \& Yennapureddy, M. K., JCAP, 02 (2018) 34. 